\title{
Movement Intention Is Better Predicted than Attention in the Posterior Parietal Cortex
}

\author{
Rodrigo Quian Quiroga, ${ }^{1}$ Lawrence H. Snyder, ${ }^{2}$ Aaron P. Batista, ${ }^{3} \mathrm{He}$ Cui, ${ }^{1}$ and Richard A. Andersen ${ }^{1}$ \\ ${ }^{1}$ Division of Biology, California Institute of Technology, Pasadena, California 91125, ${ }^{2}$ Department of Anatomy and Neurobiology, Washington University, \\ St. Louis, Missouri 63130, and ${ }^{3}$ Department of Electrical Engineering, Stanford University, Stanford, California 94305
}

We decoded on a trial-by-trial basis the location of visual targets, as a marker of the locus of attention, and intentions to reach and to saccade in different directions using the activity of neurons in the posterior parietal cortex of two monkeys. Predictions of target locations were significantly worse than predictions of movement plans for the same target locations. Moreover, neural signals in the parietal reach region (PRR) gave better predictions of reaches than saccades, whereas signals in the lateral intraparietal area (LIP) gave better predictions of saccades than reaches. Taking together the activity of both areas, the prediction of either movement in all directions became nearly perfect. These results cannot be explained in terms of an attention effect and support the idea of two segregated populations in the posterior parietal cortex, PRR and LIP, that are involved in different movement plans.

Key words: attention; motor intention; single-trial analysis; population coding; vision; parietal

\section{Introduction}

Reaching to an object or shifting gaze to a certain location involves sensorimotor transformations from visual inputs to motor planning and execution. Converging evidence has shown that the posterior parietal cortex (PPC) is a key node in this process, being involved in different types of movement intentions (Andersen and Buneo, 2002). In fact, the PPC lies between the primary visual areas in the occipital lobe and the motor cortex, thus having a privileged location for visuomotor transformations. Studies of the PPC have documented an anatomical specialization for different action planning: the lateral intraparietal area (LIP) for saccades (Mountcastle et al., 1975; Andersen et al., 1987; Gnadt and Andersen, 1988; Barash et al., 1991; Mazoni et al., 1996; Snyder et al., 1997, 1998, 2000; Zhang and Barash, 2000; Batista and Andersen, 2001; Andersen and Buneo, 2002; Dickinson et al., 2003), the parietal reach region (PRR) for reaches (Snyder et al., 1997, 1998, 2000; Batista and Andersen, 2001; Andersen and Buneo, 2002; Calton et al., 2002), and the anterior intraparietal area for grasping (Sakata et al., 1995).

Because it is also well known that PPC neurons respond to visual stimuli, some studies have focused on the modulation of sensory-related activity by attention (Robinson et al., 1978; Bushnell et al., 1981) and asserted that PPC does not play a role in movement planning (Colby and Goldberg, 1999). More recent studies focusing on LIP in PPC have drawn a similar conclusion (Goldberg et al., 1990, 2002; Gottlieb et al., 1998; Colby and

\footnotetext{
Received Aug. 16, 2005; revised Feb. 12, 2006; accepted Feb. 13, 2006.

This work was supported by the Sloan-Swartz Foundation, National Institutes of Health, Defense Advanced Research Projects Agency, Office of Naval Research, and the Boswell Foundation.

Correspondence should be addressed to Richard A. Andersen., Division of Biology, California Institute of Technology, MC 216-76, Pasadena, CA 91125. E-mail: andersen@vis.caltech.edu.

R. Quian Quiroga's present address: Department of Engineering, University of Leicester, Leicester LE1 7RH, UK. DOl:10.1523/JNEUROSCI.3468-05.2006

Copyright $\odot 2006$ Society for Neuroscience $\quad 0270-6474 / 06 / 263615-\bullet \$ 15.00 / 0$
}

Goldberg, 1999; Gottlieb and Goldberg, 1999; Kusunoki et al., 2000; Powell and Goldberg, 2000; Bisley and Goldberg, 2003). The argument is made that the sensory activity and attentionrelated activations would confound any movement planning signals (Bisley and Goldberg, 2003). These assertions are made from the perspective of single LIP neurons, in which the encoding of two parameters, attention and intention, can both contribute to the firing rate of a single cell. However, the brain codes information in the activity of populations of cells, and thus it is possible that more than a single parameter can be read out within a particular neural ensemble.

In the present study, we ask whether it is possible to predict the locus of attention from a population of PPC neurons and whether, from the same neurons, it is possible to predict different movement plans on a single-trial basis. If PPC cells reflect only the response to visual inputs and attention to the target location, as suggested by the attention-only argument (Bisley and Goldberg, 2003), then we expect that the prediction of the two movements will be confused. This should compromise the decoding performance, and it should only be possible to predict the target location and not the movement plan. If instead we can reliably predict the actual movements, this will provide conclusive evidence for a role of PPC in movement planning.

\section{Materials and Methods}

Behavioral tasks and recordings. Responses of neurons in the PPC of two monkeys were studied in interleaved delayed reach and saccade trials. In the delay trials, monkeys were briefly cued to a particular location but had to withhold the movement, which was only executed after a Go signal. For the first monkey, 47 PRR and 32 LIP cells were recorded from the left hemisphere, and, for the second monkey, 24 PRR and 39 LIP cells were recorded from the right hemisphere. Cells from the first monkey as well as the PRR cells and 11 LIP cells of the second monkey were reported previously by Snyder et al. (1997). The remaining 28 LIP cells of the second monkey are a result of new recordings. 
Target locations for reaches and saccades were marked with eight buttons surrounding a central fixation point. Buttons contained a red and a green light-emitting diode (LED). They had a diameter of $3.7 \mathrm{~cm}$ and were distributed $7.5 \mathrm{~cm}$ apart in a $3 \times 3$ matrix placed in a vertical board at $28 \mathrm{~cm}$ from the monkeys. Trials started with an initial fixation $\left( \pm 2.7^{\circ}\right)$ and a depression with the contralateral hand of the illuminated central button. After $750 \mathrm{~ms}$, one of the eight possible targets was flashed for 150 $\mathrm{ms}$ with either a green or red light, marking a delayed-reach or delayedsaccade trial, respectively. Then, after 1-1.6 s, the central LED extinguished (Go signal), and the monkey had either to saccade or reach to the remembered peripheral location while maintaining the central button depression in the first case and central fixation in the latter. Delayedsaccade and delayed-reach trials to the eight possible directions were randomly interleaved.

We also studied a dissociation task, which was very similar to the delay conditions but, in this case, after the initial fixation period, one red and one green target appeared simultaneously in opposite directions (Snyder et al., 1997). The monkey had to remember the position of the targets during the memory period, and, after the Go signal, he had to make a saccade to the previously cued red location and a reach to the previously cued green location. In this case, only preferred and nonpreferred directions were tested. For the first monkey, we recorded 28 cells in LIP and 21 in PRR performing delayed reaches, delayed saccades, and dissociation trials to the preferred and nonpreferred directions. For the second monkey, we recorded 25 cells in LIP and 27 in PRR. Experimental procedures were in accordance with the guidelines of the California Institute of Technology Institutional Animal Care and Use Committee.

Data analysis. Except for the dissociation task, all cells had a total of 128 correctly performed trials, namely eight trials for each of the eight tested directions and for the two movement types (delayed reaches and delayed saccades). For the dissociation task, only the preferred and nonpreferred directions were tested, and therefore we had a total of 48 trials: eight trials $\times$ two directions $\times$ three movement types (delayed reaches, delayed saccades, and dual movements). For each cell and trial, the mean firing rate between 150 and $750 \mathrm{~ms}$ after stimulus onset was used as input to the decoding algorithm. Cells were considered as simultaneously recorded in the sense that, for each of the eight trials (for a given movement and direction), the responses of all cells were grouped together as a single trial with $m$ values (in which $m$ is the number of cells). Trials were considered as points in an $m$-dimensional space, each coordinate representing the mean firing rate for each of the $m$ cells. One at a time, each trial was predicted based on the distribution of all other trials (leave-oneout decoding) and was assigned to the class of its nearest neighbor in the $m$-dimensional space, using Euclidean distance (Duda et al., 2001).

Figure 1 summarizes this procedure. Say we have two PRR cells, A and $\mathrm{B}$, the first cell with a preferred direction upward and the second one with a preferred direction downward. For simplicity, let us consider only these two directions. Figure $1 A$ shows the responses of both cells for a given trial to be decoded. Figure $1 B$ shows the representation of this trial as a single point (in green) in the corresponding two-dimensional space. All the other points correspond to reach-up (blue) and reach-down (red) trials. The trial to be decoded is assigned to its nearest neighbor, i.e., a reach-up (blue) trial. Decoding results are displayed as "confusion matrices," in which the values on a given row $i$ and column $j$ of the matrix represent the (normalized) number of times a given "true direction" $i$ is predicted to be $j$. Note that the sum of each row equals 1 . If the decoding is perfect, $i=j$ for all trials, and the confusion matrix will show a diagonal with 1 and the rest will be 0 (left matrix). The mean decoding performance is defined as the average across the diagonal ( 1 in the case of perfect decoding). If decoding is at chance, each decoding direction is equally likely, and the decoding performance will be $1 / 8=0.125$.

Statistical analysis. For assessing statistical significance of the decoding results, two types of tests were performed. First, we compared the decoding of reach plans, saccade plans, and target locations. For each decoding, a sequence of $n$ outcomes (in which $n$ is the number of trials) was generated by assigning a value of 1 to correctly predicted trials and a value of 0 to the incorrect ones. Then, significance was established by comparing these sequences (e.g., decoding of reaches vs decoding of saccades) using a nonparametric Wilcoxon's rank test. Because the number of cells in

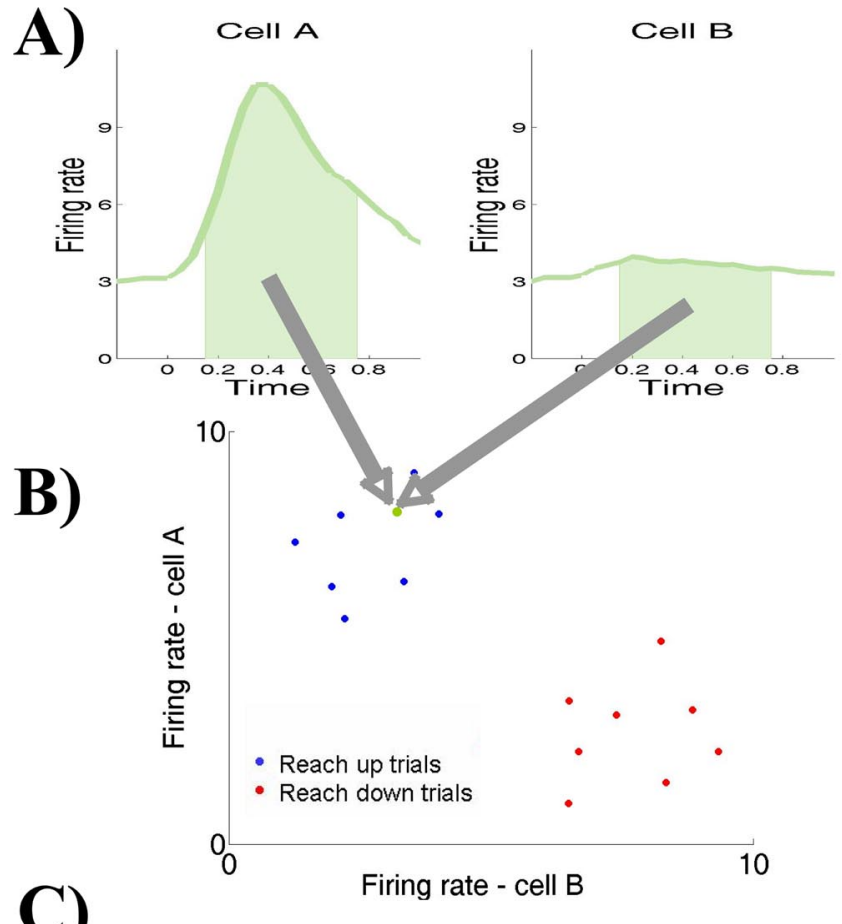

C)

\section{Perfect decoding}

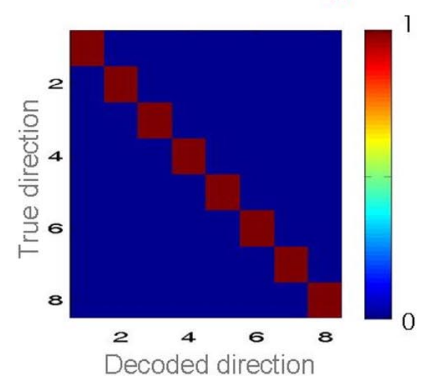

Chance decoding

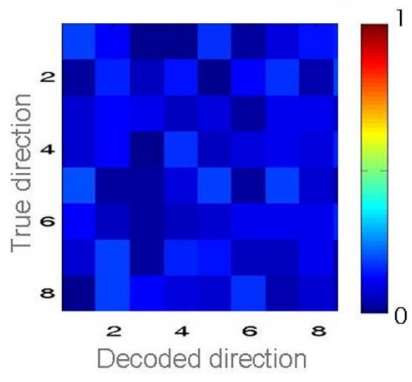

Figure 1. Outline of the decoding procedure. $\boldsymbol{A}, \boldsymbol{B}$, For a given trial to be decoded, the mean firing rate between 150 and 750 ms after stimulation is represented as a point in an $m$-dimensional space (in green; $m$ is the number of neurons). The trial is assigned to the class of its nearest neighbor (blue, reach-up trial). $C$, If decoding is perfect, the confusion matrix will show a diagonal with 1 and the rest of the matrix 0 . If decoding is at chance, each decoded direction is equally likely.

PRR is different from the number in LIP and because they have different spatial tunings, all comparisons were done using the same cell populations. In particular, when comparing the decoding of reach plans, saccade plans, and target locations, we first used the population of PRR cells, then the population of LIP cells, and then both populations together.

Second, we established whether each decoding performance was significantly better than chance by comparing the sequences of decoding outcomes with "chance sequences," in which correct and incorrect trials were randomly assigned with a probability given by the chance level (i.e., for eight directions, a correct trial is assigned with a probability of $1 / 8$ ).

\section{Results}

\section{Single-cell responses}

Figure 2 shows the response to delayed-reach and delayedsaccade trials in eight different directions for a typical cell in PRR $(A)$ and for another typical cell in LIP $(B)$. The PRR cell shows a clear increase in firing rate with reaches to the upper and the upper right directions. In the case of the LIP cell, the maximum increase in firing rate is for saccades to the upper right. Note that these activations are in the delay period, i.e., after target offset and before the execution of the movement, and are therefore not a 


\section{A) \\ PRR cell}
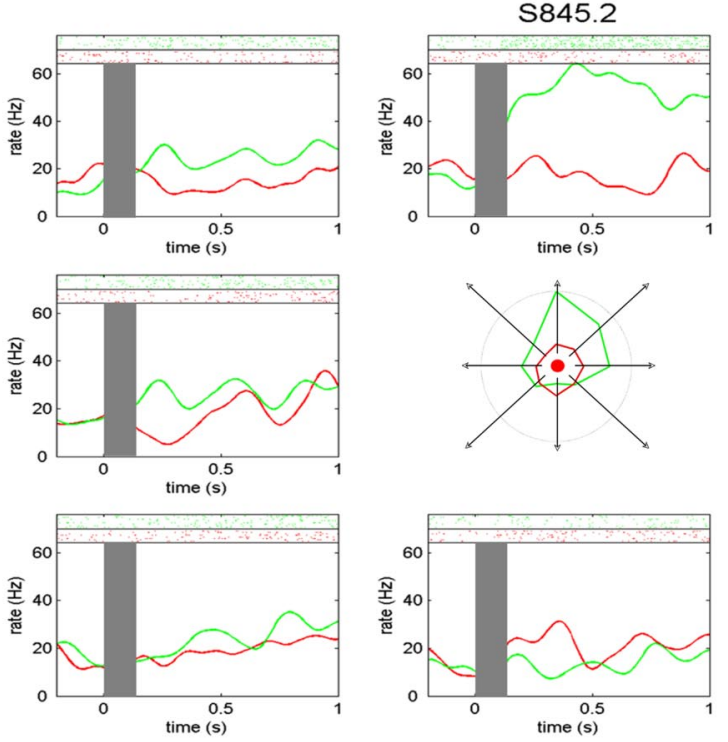

B)

LIP cell
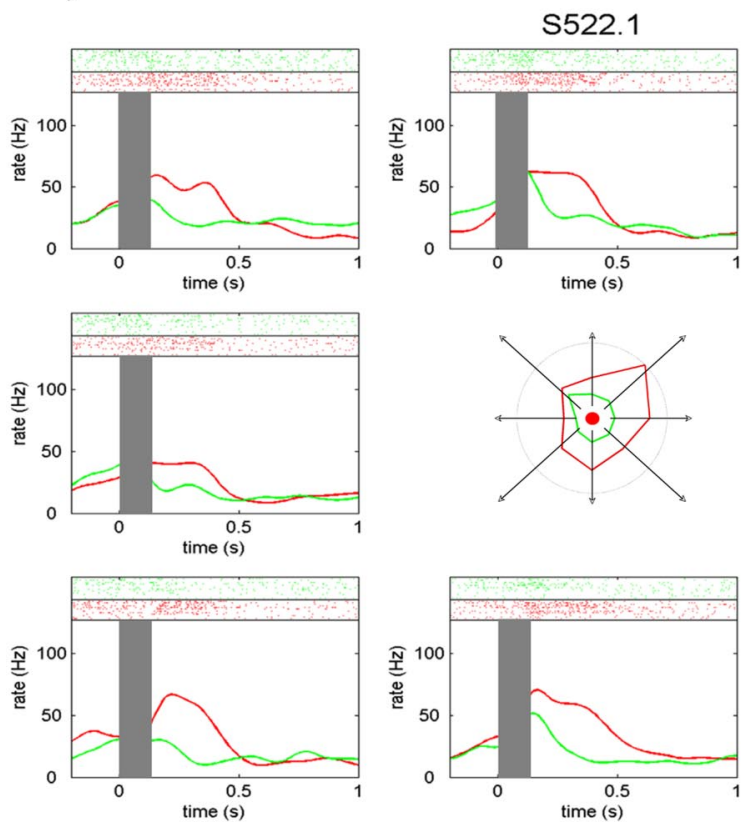
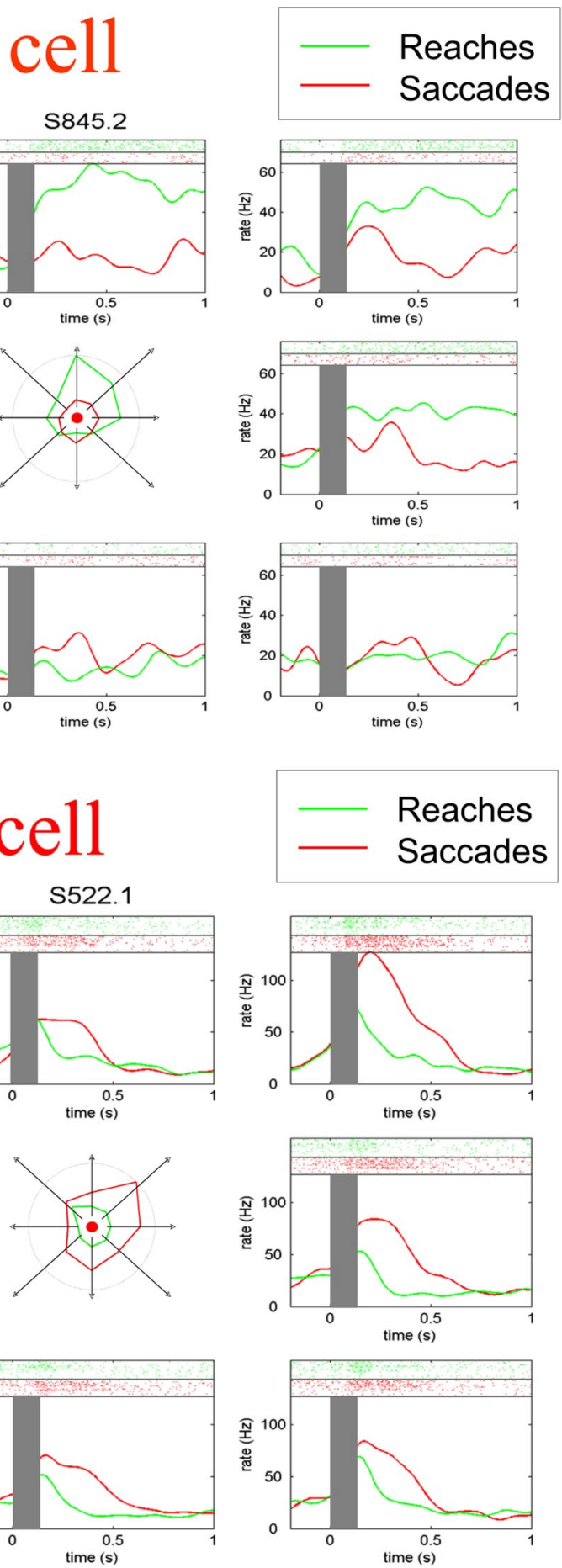

Figure 2. Responses of a PRR cell $(\boldsymbol{A})$ and an LIP cell $(\boldsymbol{B})$ to delayed-reach and delayed-saccade trials. Each plot shows the response to a different direction, with the raster plots in the top part and the mean firing rates in the bottom. Gray rectangles mark the time of target presentation. The center insets show the spatial tunings of the cells in the time period considered (150-750 ms). Note the tuning for reaches (green) to the upper direction for the PRR cell and the tuning for saccades (red) to the upper right direction for the LIP cell.

result of the target presentation or the actual movement. To a lesser degree, there is also spatial tuning to the nonpreferred movements, i.e., saccades for the PRR cell and reaches for the LIP cell. In principle, this spatial tuning may indicate that these cells signal the locus of attention (i.e., the place where the target was shown) rather than movement intentions. In fact, taking the activity of single cells alone, it seems difficult to predict motor intentions because reaches and saccades may be confused.

\section{Decoding of target locations}

We first studied the possibility of predicting target locations as would be favored by the attention-only argument. Indeed, if the responses of cells in PPC are solely attributable to attention, then it should not matter what movement is being planned. To study this possibility, we pooled together delayed-reach and delayed-saccade trials and grouped them according to target location. Figure 3 shows the decoding of target locations analyzing PRR (left matrix) and LIP (right matrix) separately and together (middle matrix) for the first monkey. In all cases, the decoding performance was significantly larger than chance $\left(p<10^{-9}\right.$ with PRR cells, $p<10^{-3}$ with LIP cells, and $p<10^{-13}$ taking both populations together). For the LIP cells, there was a significantly better decoding of the contralateral $(0.50)$ than the ipsilateral $(0.16 ; p<0.05)$ field. For the PRR cells, there were no significant differences between the ipsilateral (0.62) and contralateral $(0.70 ; p=0.55)$ fields.

Results for the second monkey were qualitatively the same. Decoding of target locations were significantly better than chance ( $p<0.005$ with PRR cells, $p<$ $10^{-4}$ with LIP cells, and $p<10^{-9}$ taking both populations together). For the LIP cells, decoding was significantly better for the contralateral $(0.58)$ than the ipsilateral $(0.21 ; p<0.01)$ field. As with the first monkey, for the PRR cells, there were no significant differences between the ipsilateral $(0.29)$ and contralateral $(0.29 ; p=1)$ fields.

\section{Decoding of reach and saccade plans}

Figure $4 \mathrm{~A}$ shows the results of decoding the delayed-reach and delayed-saccade trials of the first monkey, considering the populations of PRR and LIP cells separately. Cells in PRR (top plots) have a nearly perfect performance in decoding reach directions $(0.90)$, with only a few errors caused by confusion of nearby directions. The performance is very good for both the ipsilateral and the contralateral directions, without a significant difference between them $(p=0.39)$. The performance of PRR cells for decoding of saccades $(0.51)$ is significantly worse than the one for reaches $\left(p<10^{-5}\right)$ but is still better than chance $\left(p<10^{-5}\right)$.

For the LIP cells, the decoding of saccades for the contralateral field $(0.87)$ is significantly better than for the ipsilateral field $\left(0.33 ; p<10^{-3}\right)$. In contrast to what is found in PRR, for LIP cells, the decoding of reaches $(0.34)$ is worse than for saccades $(0.57 ; p<0.01)$. The performance of LIP cells for decoding the "nonpreferred movement" (i.e., reaches) is also larger than chance $(p<0.005)$. Thus, these two anatomically segregated cell 
populations are involved in the planning of different movements, reaches in the case of PRR and saccades for LIP. However, this segregation of tasks for both cell populations is overlapping to some extent, and the decoding of the nonpreferred movement is larger than chance. This is in agreement with the tunings to the nonpreferred movements shown in Figure 2 for the exemplary LIP and PRR cells.

Using both cell populations for decoding reaches and saccades clearly increases the decoding performance, as seen in Figure $4 \mathrm{~B}$. The overall decoding performance is 0.84. In particular, the decoding of reaches is perfect (1.00) and in no case is a reach confused with a saccade or vice versa. The decoding of saccades is also perfect in the contralateral directions (1.00). The decoding of saccades in the ipsilateral field is poor (0.33) because of the lack of cells tuned to these saccade directions. The increase in decoding performance when using both cell populations together is attributable to the fact that PRR and LIP cells show some coding of their nonpreferred movement (reaches for LIP and saccades for PRR), which, rather than confusing the decoder, increases its power of prediction.

Results for the PRR neurons from the second monkey were qualitatively similar to those described from the first. The decoding of reaches $(0.66)$ was better than saccades $\left(0.36 ; p<10^{-3}\right)$, and, in both cases, the performance was better than chance $(p<$ $10^{-9}$ for reaches and $p<0.005$ for saccades). As with the previous monkey, for PRR cells, there were no significant differences between the ipsilateral and contralateral locations.

For the LIP cells from the second animal, there were, in principle, no significant differences between reaches (0.66) and saccades $(0.75 ; p=0.25)$. However, in this case, the decoding of saccades is significantly better in the contralateral field ( 0.92 for contralateral vs 0.62 for ipsilateral; $p<0.05$ ), and, when comparing decodings in the contralateral field, the difference between saccades and reaches was significant $(0.92$ for saccades and 0.54 for reaches; $p<0.005)$. For LIP cells, the decoding performances were also better than chance $\left(p<10^{-13}\right.$ for saccades and $p<$ $10^{-10}$ for reaches). Using both cell populations for decoding both reaches and saccades, the overall performance was 0.84 , similar to the finding for the first monkey. In particular, the decoding performance was 0.80 for saccades and 0.87 for reaches.

\section{Decoding attention versus movement intention}

In the previous sections, we showed that it is possible to predict both target location and movement plans from the activity of PPC neurons. To further understand the role of PPC cells in movement plans and attention, we compared both types of predictions. For the first monkey, with PRR cells, the decoding of the preferred movement (reaches; 0.90 ) was significantly better than the one of target locations $\left(0.62 ; p<10^{-3}\right)$, and, for LIP cells, the decoding of the preferred movement (saccades; 0.57 ) was also significantly better than the one of target locations $(0.31 ; p<$ $0.01)$. The performances for the decoding of the nonpreferred movements were comparable with the ones obtained for target locations ( $p=0.21$ for PRR and $p=0.7$ for LIP). In fact, it seems reasonable to assume that the tunings to the nonpreferred plan shown in Figure 2 may be in part related to an attention effect.

Taking both cell populations together, the prediction of

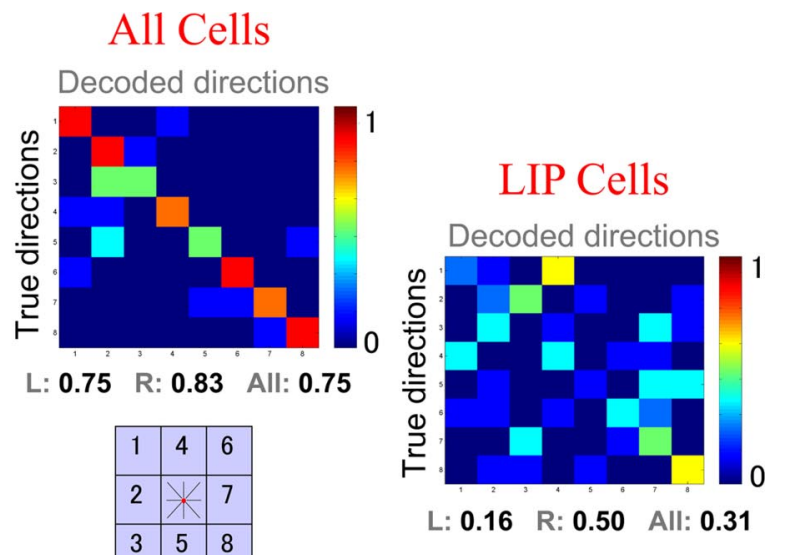


decoding of the ipsilateral target locations $(0.75 ; p<0.01)$ because, as already mentioned, PRR cells code information regarding both hemifields.

Reinforcing these results, we note that, in principle, it should have been statistically easier to predict target locations ( 1 of 8 choices) than the actual movement and its direction ( 1 of 16 choices). The better performance in decoding movement plans than target locations is attributable to the differential response of PRR and LIP cells to reaches and saccades. In other words, for PRR cells, the response to reaches to the preferred direction is much stronger than the response to saccades to the same location (and the opposite for LIP) (Fig. 2). Therefore, if the actual movement is not considered, the same cell switches from high to low responses for the same preferred target location, thus confusing the decoding.

We also considered differences between the decoding of target locations and movement intentions using the cue period (i.e., we used the mean number of spikes between 0 and $150 \mathrm{~ms}$ after stimulus onset as inputs to the decoding algorithm). In this case, all of the differences described above did not reach significance. Thus, visual sensory responses, and possibly transient shifts of attention, produce similar responses during the cue period.

Results for the second monkey were qualitatively the same. In fact, for PRR cells, the decoding of the preferred movement (reaches; 0.66 ) was significantly better than the one of target locations $\left(0.36 ; p<10^{-3}\right)$. For LIP cells, the decoding of the preferred movement (saccades; 0.75$)$ was also significantly better than the one of target locations $\left(0.44 ; p<10^{-4}\right)$. Taking both cell populations together, the decoding of movement intentions (reaches and saccades; 0.84 ) was significantly better than the one of target location $(0.69 ; p<0.05)$

\section{Dual-movement task}

Next we considered the dual-movement experiment, in which, after a delay period, the monkey had to make a saccade and a reach to previously cued opposite directions. Figure $5 A$ shows the results for a typical cell in PRR and another one in LIP from the first monkey. For the PRR cell, there is a large activation for reaches to the preferred direction, with also some activation to saccades. Because reaches to the preferred direction are accompanied by saccades to the nonpreferred direction (and vice versa), for the PRR cell, we took as the preferred direction for the dissociation trials the one given by the reaches. For the LIP neuron, the preferred direction is the one given by the saccades. Note that, in the dissociation task, the firing of the PRR cell during saccades to the preferred direction (and reaches to the nonpreferred) is lower than the one during saccades to the preferred direction alone.

For the LIP cell, there is a fairly similar activation during delayed reaches and delayed saccades to the preferred location. However, in the dissociation task, the cell is activated only with saccades to the preferred direction. Therefore, during the dissociation task, LIP cells code for saccade plans regardless of reach plans and PRR cells code for reaches regardless of saccade plans. This is not consistent with the attention argument, which predicts similar responses to both directions in the dissociation trials and reinforces the idea of two independent populations coding for reaches and saccades, respectively.

Finally, we decoded reaches, saccades, and dissociation trials to the preferred and nonpreferred directions using the PRR and LIP populations separately and then taking them together. The overall performance using PRR cells alone was 0.79 , with most errors produced by the confusion of delayed reaches with dissociation trials having a reach to the same direction. This shows
A)

Preferred direction

Non-Preferred direction
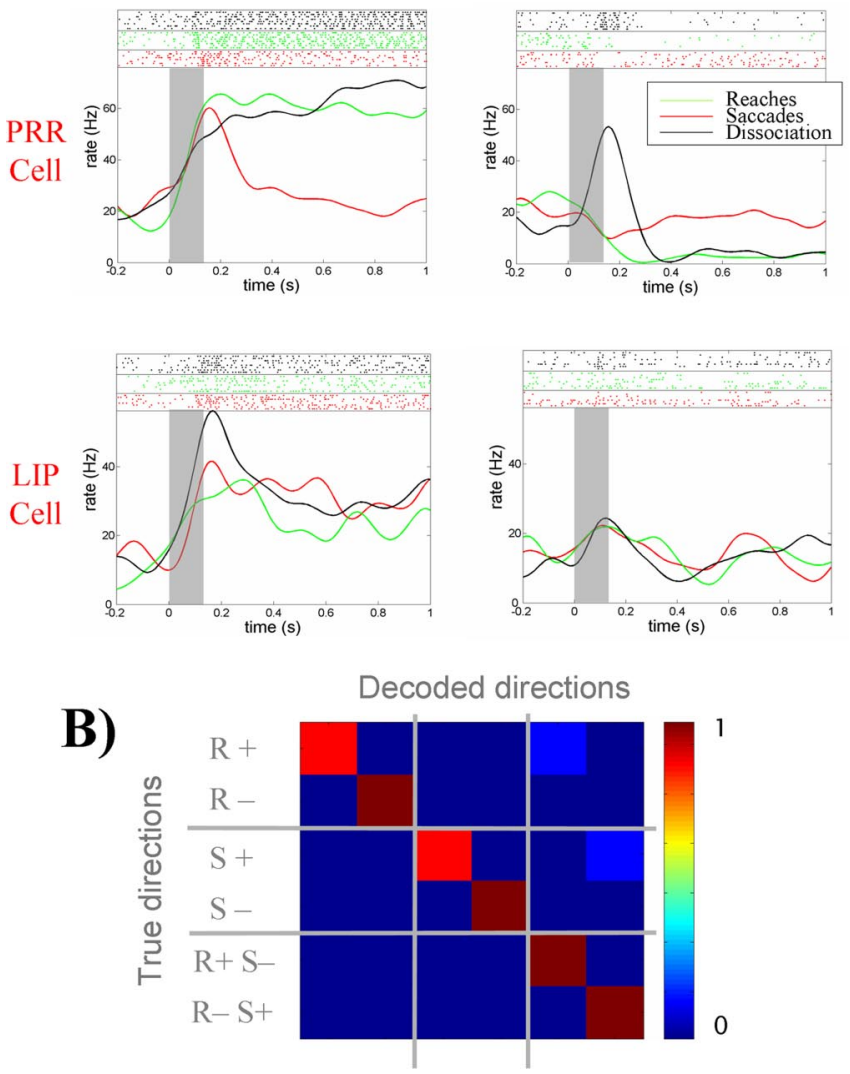

Figure 5. A, Responses of a single cell in PRR and one in LIP to delayed-reaches delayedsaccade, and dissociation trials. In dissociation trials, the saccades and reaches are performed simultaneously to opposite directions. The top parts show the raster plots, and the bottom ones show the mean firing rates. Gray rectangles mark the time of target presentation. Data for dissociation trials are plotted according to the direction of the reach for the PRR neuron and for the saccade for the LIP neuron. Note, for the dissociation task, that the PRR cell tends to follow the behavior during delayed reaches, ignoring the saccades to the opposite direction. In contrast, the LIP cell tends to follow the saccades, ignoring the reaches to the opposite direction. $\boldsymbol{B}$, The decoding of reaches $(R)$, saccades $(S)$, and dissociation trials $(R S)$ to the preferred $(+)$ and nonpreferred directions $(-)$ is nearly perfect using both LIP and PRR populations.

that, during the dissociation trials, PPC cells primarily code for the preferred movement and there is nearly no information about the nonpreferred one. The decoding with the LIP cells was 0.58 , and again most errors were attributable to the confusion of delayed saccades, with dissociation trials having a saccade to the same direction. However, this confusion was resolved when considering both populations together, and the decoding became close to perfect (0.96) (Fig. 5B). For the second monkey, results were qualitatively similar, with a performance of 0.66 using PRR cells, 0.64 using those in LIP, and 0.79 using both groups together.

\section{Discussion}

We examined at the population level whether it is possible to decode the location of visual targets, as an indicator of the locus of attention, and whether such decoding will improve when considering the actual movement plan (either a reach or a saccade). If the responses of cells in PPC are solely attributable to attention, then it should not matter what movement is being planned. We showed that predictions of target locations are better than chance, but, in contrast to previous claims (Goldberg et al., 2002; 
Bisley and Goldberg, 2003), we also showed that it is possible to reliably decode reach and saccade intentions to different locations using cells from the posterior parietal cortex. Moreover, considering only target location confused the decoding, and its performance was significantly worse than the one for movement intentions. Furthermore, PRR activity allows for a better decoding of reaches than saccades, and the opposite is true for LIP cells. Interestingly, with PRR cells, it was possible to obtain a nearly perfect decoding of reaches both for the contralateral and ipsilateral sites. For the LIP cells, the decoding was optimal only for saccades to the contralateral sites. Both PRR and LIP cells also showed some coding of their nonpreferred movement, and the best decoding results were obtained when considering both populations together. Therefore, the neural responses to the nonpreferred movements described in Figure 2, rather than confusing the predictions of movement plans, dramatically increased the power of prediction. We stress that we do not claim that PPC cells are not modulated by attention at all. In fact, the responses to the nonpreferred movements may be attributable to an attention effect.

We also show that it was possible to decode simultaneous movement plans in a dissociation task. Taking both PRR and LIP populations together, the decoding of single reaches, single saccades, and simultaneous reaches and saccades to opposite directions was nearly perfect. This gives additional strong evidence against the attention-only argument. In fact, if PPC cells are mostly driven by attention, then there should be nearly no difference when the monkey performs the two different dissociation trials because, in both cases, two targets are simultaneously flashed in the preferred and nonpreferred directions. This task requires the coordination of hand and eye movements in a more complex behavior, which, in principle, would appear much harder to decode unless both movements are primarily represented by independent cell populations. The decoding performance should be at chance if PRR and LIP cells do not carry information of the movement plans, which is clearly not the case.

In summary, the activity of PPC neurons is more predictive of the impending movement than the locus of attention. Such predictions improve when an entire population of PPC neurons is considered. This evidence conclusively shows the existence of two segregated brain areas in the PPC involved in different movement plans.

\section{References}

Andersen RA, Buneo CA (2002) Intentional maps in posterior parietal cortex. Annu Rev Neurosci 25:189-220.

Andersen RA, Essick GK, Siegel RM (1987) Neurons of area 7a activated by both visual stimuli and oculomotor behavior. Exp Brain Res 67:316-322.

Barash S, Bracewell RM, Fogassi L, Gnadt JW, Andersen RA (1991) Saccaderelated activity in the lateral intraparietal area II. Spatial properties. J Neurophysiol 66:1109-1124.

Batista AP, Andersen RA (2001) The parietal reach region codes the next planned movement in a sequential reach task. J Neurophyisol 85:539-544.

Bisley JW, Goldberg ME (2003) Neuronal activity in the lateral intraparietal area and spatial attention. Science 299:81-86.

Bushnell MC, Goldberg ME, Robinson DL (1981) Behavioral enhancement of visual responses in monkey cerebral cortex. I. Modulation in posterior parietal cortex related to selective visual attention. J Neurophysiol 46:755-772.

Calton JL, Dickinson AR, Snyder LH (2002) Non-spatial, motor-specific activation in posterior parietal cortex. Nat Neurosci 5:580-588.

Colby CL, Goldberg ME (1999) Space and attention in parietal cortex. Annu Rev Neurosci 22:319-349.

Dickinson AR, Calton JL, Snyder LH (2003) Non-spatial saccade-specific activation in area LIP of monkey parietal cortex. J Neurophysiol 90:2460-2464.

Duda OH, Hart PE, Stork DG (2001) Pattern classification, Ed 2. New York: Wiley.

Gnadt JW, Andersen RA (1988) Memory related motor planning activity in posterior parietal cortex of macaque. Exp Brain Res 70:216-220.

Goldberg ME, Colby CL, Duhamel JR (1990) Representation of visuomotor space in the parietal lobe of the monkey. Cold Spring Harb Symp Quant Biol 55:729-739.

Goldberg ME, Bisley J, Powell KD, Gottlieb J, Kusunoki M (2002) The role of the lateral intraparietal area of the monkey in the generation of saccades and visuospatial attention. Ann NY Acad Sci 956:205-215.

Gottlieb J, Goldberg ME (1999) Activity of neurons in the lateral intraparietal area of the monkey during an antisaccade task. Nat Neurosci 2:906-912.

Gottlieb JP, Kusunoki M, Goldberg ME (1998) The representation of visual salience in monkey parietal cortex. Nature 391:481-484.

Kusunoki M, Gottlieb J, Goldberg ME (2000) The lateral intraparietal area as a salience map: the representation of abrupt onset, stimulus motion, and task relevance. Vision Res 40:1459-1468.

Mazoni P, Bracewell RM, Barash S, Andersen RA (1996) Motor intention activity in the macaque's lateral intraparietal area. I. Dissociation of motor plan from sensory memory. J Neurophysiol 76:1439-1456.

Mountcastle VB, Lynch JC, Georgopoulos A, Sakata H, Acuna C (1975) Posterior parietal association cortex of the monkey: command functions for operations within extrapersonal space. J Neurophysiol 38:871-908.

Powell KD, Goldberg ME (2000) Response of neurons in the lateral intraparietal area to a distractor flashed during the delay period of a memoryguided saccade. J Neurophysiol 84:301-310.

Robinson DL, Goldberg ME, Stanton GB (1978) Parietal association cortex in the primate: sensory mechanisms and behavioral modulations. J Neurophysiol 41:910-932.

Sakata H, Taira M, Murata A, Mine S (1995) Neural mechanisms of visual guidance of hand action in the parietal cortex of the monkey. Cereb Cortex 5:429-438.

Snyder LH, Batista AP, Andersen RA (1997) Coding of intention in the posterior parietal cortex. Nature 386:167-170.

Snyder LH, Batista AP, Andersen RA (1998) Change in motor plan, without a change in the spatial locus of attention, modulates activity in posterior parietal cortex. J Neurophysiol 79:2814-2819.

Snyder LA, Batista AP, Andersen RA (2000) Intention-related activity in the posterior parietal cortex: a review. Vision Res 40:1433-1441.

Zhang M, Barash S (2000) Neuronal switching of sensorimotor transformations for antisaccades. Nature 408:971-975. 